BULLETIN OF THE

AMERICAN MATHEMATICAL SOCIETY

Volume 78, Number 4, July 1972

\title{
A REPRESENTATION OF A POSITIVE LINEAR MAPPING
}

\author{
BY OHOE KIM ${ }^{1}$
}

Communicated by Dorothy Stone, January 4, 1972

Let $X$ and $Y$ be compact Hausdorff spaces. Let $C(X)$ and $C(Y)$ be the algebras of real valued continuous functions on $X$ and $Y$ respectively. $C(X)$ and $C(Y)$ are endowed with their natural partial ordering and their sup norm. Let $\Phi: C(X) \rightarrow C(Y)$ be a positive, bounded linear mapping.

$X$ is said to have the Souslin property if every disjoint family of nonempty open subsets of $X$ is countable.

A lattice $L$ is said to satisfy the countable chain condition upward if the following is true: For any upper bounded subset $A$ of $L$, there exists a countable subset $B$ of $A$ such that $A$ and $B$ have the same family of upper bounds. The countable chain condition downward on a lattice can be defined in a similar fashion.

A lattice $L$ is said to satisfy the countable chain condition if $L$ satisfies both the countable chain condition upward and the countable chain condition downward.

The purpose of this note is to announce the results on representation for $\Phi$, based on the techniques developed in [1], [2].

To get the main theorem, we need the following series of propositions which are interesting in themselves.

Proposition. For a given compact Hausdorff space $X$, there exists a complete Boolean space $X^{*}$ and a mapping $\sigma: C(X) \rightarrow C\left(X^{*}\right)$ such that $\sigma$ is an isometric, order preserving and algebra monomorphism.

Remark. The construction of $\sigma$ here is different from the one in [3]. A part of the proof comes from an application of the Gelfand-Naimark theorem [4].

We study a necessary and sufficient condition on $X$ under which $C\left(X^{*}\right)$ satisfies the countable chain condition so that we later use this result to represent $\Phi$ as the Maharam integral [2].

To this end, we introduce the concept of the countable chain condition on a Boolean algebra [6] and the pseudocountable chain condition on $C(X)$.

$C(X)$ is said to satisfy the pseudocountable chain condition if every disjoint set of nonzero elements of $C(X)$ is countable. (Two functions $f$ and $g$ of $C(X)$ are disjoint if inf $(f, g)=0$.)

AMS 1970 subject classifications. Primary 46E15, 47B55, 28A40.

Keywords and phrases. Positive mapping, the Souslin property, representation, the Maharam integral.

${ }_{1}$ The work announced here is a part of the author's doctoral thesis at the University of Rochester under the supervision of Professor D. Maharam Stone, to whom he wishes to express his warm thanks. 
Proposition. $X$ has the Souslin property if and only if $C\left(X^{*}\right)$ has the countable chain condition.

REMARK. The proof goes roughly as follows: First we show that the countable chain condition on $C\left(X^{*}\right)$, the pseudocountable chain condition on $C\left(X^{*}\right)$ and the Souslin property on $X^{*}$ are all equivalent. Next, we show that $X^{*}$ has the Souslin property if and only if $X$ has the Souslin property.

We are concerned with an extension of $\Phi$. Let $K(X)$ and $K(Y)$ be the spaces of Baire functions on $X$ and $Y$ respectively. In [5], it was shown that $K(X)$ and $K(Y)$ contain $C(X)$ and $C(Y)$ respectively.

Proposition. There is a unique extension $\Phi_{1}: K(X) \rightarrow K(Y)$ of $\Phi$ with $\left\|\Phi_{1}\right\|=\|\Phi\|$. Furthermore, $\Phi_{1}$ is a positive, linear and countably additive mapping.

Finally, we have the following theorem.

THEOREM. Let $X$ and $Y$ have the Souslin property. Then $\Phi$ can be expressed as the Maharam integral.

Remark 1. For the definition of the Maharam integral, we refer to [2]. Roughly, we may rephrase the theorem as follows. Under the above assumptions on $X$ and $Y$, there exist compact Hausdorff spaces $R$ and $S$ such that $C\left(X^{*}\right)$ is "isomorphic" to a certain space of functions on $R \times S$ and $C\left(Y^{*}\right)$ is isomorphic to a space of functions on $R$, and under these isomorphisms, $\Phi$ corresponds to the mapping $f \mapsto f^{\prime}$ where $f^{\prime}(r)$ $=\int_{s} f(r, s) d \mu$, the integral being formed with respect to an ordinary $\sigma$-finite numerical measure $u$ on $S$.

REMARK 2. The proof relies on the preceding propositions and the techniques e.g., a direct product $J \otimes U$ in Maharam's sense, developed in [1], [2] to get a generalized form of the Maharam integral. To complete the proof, it is necessary to realize a certain set mapping as a point mapping.

Detailed proofs and applications of these results will appear elsewhere.

\section{REFERENCES}

1. D. Maharam, The representation of abstract measure functions, Trans. Amer. Math. Soc. 65 (1949), 279-330. MR 10, 519.

2. , The representation of abstract integrals, Trans. Amer. Math. Soc. 75 (1953), 154-184. MR 14 \# 1071.

3. A. M. Gleason, Projective topological spaces, Illinois J. Math. 2 (1958), 482-489. MR 22 \#12509.

4. I. M. Gel'fand and M. A. Naìmark, On the embedding of normed rings into the ring of operators in Hilbert space, Mat. Sb. 12 (54) (1943), 197-213. MR 5, 147.

5. P. R. Halmos, Measure theory, Van Nostrand, Princeton, N.J., 1950. MR 11, 504. 6. - Lectures on Boolean algebras, Van Nostrand Math. Studies, no. 1, Van Nostrand, Princeton, N.J., 1963. MR 29 \#4713.

Department of Mathematics, Carnegie-Mellon University, Pittsburgh, PennSYLVANIA 15213. 